\title{
Getting a Low Grade for Missing High-Grade Dysplasia and Colorectal Cancer in IBD
}

\author{
James R. Conner ${ }^{1} \cdot$ Robert H. Riddell ${ }^{1}$
}

Received: 5 October 2017/ Accepted: 7 October 2017/Published online: 20 October 2017

(C) Springer Science+Business Media, LLC 2017

Inflammatory bowel disease (IBD) is associated with an increased risk of developing colorectal cancer (CRC) associated with the duration and extent of the disease [1]. Unlike sporadic CRC, CRC in IBD patients is often difficult to detect, due to the presence of ill-defined margins and often atypical gross and endoscopic appearances [2]. CRC in IBD patients is associated not only with high-grade dysplasia (HGD) but also with low-grade dysplasia (LGD), either of which may be multifocal, polypoid, flat, or invisible. Accordingly, surveillance at 2-year intervals is recommended starting 8-10 years after diagnosis, with colectomy recommended for colonoscopic identification of high-risk lesions such as multifocal or polyploid LGD, HGD, and CRC [2]. Over the last decade, as newer endoscopic techniques such as chromo-, narrow-band, and highdefinition endoscopy have matured, the management of IBD-associated mucosal abnormalities, particularly LGD, has shifted toward decreasing rates of colectomy in favor of endoscopic management [3]. This trend was generally endorsed in the Surveillance for Colorectal Endoscopic Neoplasia Detection and Management in Inflammatory Bowel Disease Patients: International Consensus Recommendation (SCENIC) guidelines for the management of dysplasia and carcinoma in IBD [4]. An important consideration as these newer strategies become more widespread is the rate of undetected advanced lesions (HGD and invasive carcinoma) in patients undergoing endoscopic surveillance, for which remarkably few data are available.

Robert H. Riddell

Robert.Riddell@sinaihealthsystem.ca

1 Departments of Pathobiology and Laboratory Medicine, Mt. Sinai Hospital, University of Toronto, 600 University Avenue, Toronto M5G 1X5, Canada
In this issue of Digestive Diseases and Sciences, Eluri et al. [5] reported a retrospective analysis of data from a single institution collected between 1994 and 2013 in subjects with IBD who had undergone colectomy in which the specimens contained HGD or CRC. Of the 70 subjects, 20 underwent colectomy for indications other than prior endoscopic identification of HGD or CRC (the "undetected group"), such as LGD, failure of medical management, and complications such as fistula formation, obstruction, and perforation. The remaining 50 subjects underwent colectomy following the colonoscopic identification of HGD or CRC (the "detected" group).

The authors point out that most patients underwent colonoscopy within 2 years of colectomy, suggesting that the HGD or carcinoma was most likely missed rather than occurring as true interval lesions. Importantly, the rate of undetected high grade dysplasia or invasive carcinoma was unchanged over the 20-year study period. Although detailed data on endoscopic methods were not available, it is certainly reasonable to assume that more advanced techniques were employed in the later years of the study.

Two patients (10\%) in the undetected group had entirely negative surveillance histories. Of the remaining $90 \%$, prior lesions were classified as LGD in $75 \%$, and indefinite for dysplasia in $15 \%$. The majority of subjects (15) in the undetected group had HGD in the resected colon without evidence of CRC. In the five cases with CRC, all patients had a history of either multifocal LGD or LGD involving an unresectable polyp or stricture; none with CRC in the resected colon had a history of only unifocal, flat, low-grade dysplasia. The advanced lesions most commonly occurred in the right colon or in the rectum.

This report provides a sobering look at the risk of undetected HGD or invasive carcinoma in patients under 
surveillance, especially given the strong association of HGD with invasive carcinoma. The persistence of missed cases into the later years of the study period indirectly challenges the notion that high-definition techniques are unlikely to miss CRC in those with LGD, which was advanced by a study in which multiple high-grade and lowgrade dysplastic lesions were also missed [6]. It is somewhat reassuring that all patients with CRC had LGD arising in preoperative lesions that, in the light of recent guidelines, would have prompted more serious consideration of colectomy (strictures, unresectable polyps, and multifocal polypoid dysplasia). Nevertheless, the risk of undetected HGD or invasive carcinoma should be carefully considered in future management recommendations. Had surgeons and pathologists formed a majority of the authors of the SCENIC guidelines, the recommendations may have been quite different.

\section{References}

1. Eaden JA, Abrams KR, Mayberry JF. The risk of colorectal cancer in ulcerative colitis: a meta-analysis. Gut. 2001;48:526-535.

2. Bae SI, Kim YS. Colon cancer screening and surveillance in inflammatory bowel disease. Clin Endosc. 2014;47:509-515.

3. Choi $\mathrm{CH}$, Rutter MD, Askari A, et al. Forty-year analysis of colonoscopic surveillance program for neoplasia in ulcerative colitis: an updated overview. Am J Gastroenterol. 2015;110:1022-1034.

4. Laine L, Kaltenbach T, Barkun A, et al. SCENIC international consensus statement on surveillance and management of dysplasia in inflammatory bowel disease. Gastroenterology. 2015;148:639-651.

5. Eluri S, Parian AM, Limketkai BN, et al. Nearly a third of highgrade dysplasia and colorectal cancer is undetected in patients with inflammatory bowel disease. Dig Dis Sci. (Epub ahead of print). doi:10.1007/s10620-017-4652-5.

6. Krugliak Cleveland N, Colman RJ, Rodriquez D, et al. Surveillance of IBD using high definition colonoscopes does not miss adenocarcinoma in patients with low-grade dysplasia. Inflamm Bowel Dis. 2016;22:631-637. 